\title{
Proteolytic Enzyme Combination Reduces Inflammation and Oxidative Stress and Improves Insulin Sensitivity in a Model of Metabolic Syndrome
}

\author{
Tetiana V. Talaieva*, Victor V. Bratus \\ National Scientific Center, ND Straschesko Institute of Cardiology, Academy of Medical Sciences of Ukraine, \\ Kiev, Ukraine \\ Email: ${ }^{*}$ talaieva-t@yandex.ru
}

Received 6 February 2015; accepted 17 February 2015; published 2 March 2015

Copyright @ 2015 by authors and Scientific Research Publishing Inc.

This work is licensed under the Creative Commons Attribution International License (CC BY). http://creativecommons.org/licenses/by/4.0/

(c) (i) Open Access

\begin{abstract}
Chronic, low-level inflammation may be an independent marker of Metabolic Syndrome (MetS). Systemic Enzyme Therapy (SET), the oral administration of proteolytic enzymes, is safe and effective in the management of inflammation. Therefore, the effects of SET, as Wobenzym ${ }^{\circledR}$, on the prevention and treatment of inflammation and other metabolic risk factors were assessed in a rabbit model of diet-induced MetS. Animals were fed a lipid-enriched diet for 8 weeks during which they were administered a vehicle control (control group) or Wobenzym either throughout the study period (prevention group) or beginning at the 5th week, after the development of biomarkers of MetS (treatment group). At the 8th week, both prevention and treatment groups demonstrated improved insulin sensitivity relative to the control group and reduced serum C-reactive protein (CRP) and glycosylated hemoglobin (HbA1c, $P<0.001$ ). At 8 weeks, the prevention group, but not the treatment group, exhibited reduced total cholesterol and oxidative stress, measured as serum malondialdehyde $(P<0.001)$. Triglycerides and free fatty acids were reduced in both the treatment $(P<0.01)$ and prevention groups $(P<0.001)$ relative to the control group at the 8th week. Body weight and blood glucose were not affected. Enzyme therapy may have a positive effect on inflammation, insulin sensitivity, and other metabolic risk factors of MetS.
\end{abstract}

\section{Keywords}

Metabolic Syndrome, Systemic Enzyme Therapy, Wobenzym, Protease, Inflammation, CRP

\footnotetext{
${ }^{*}$ Corresponding author.
}

How to cite this paper: Talaieva, T.V. and Bratus, V.V. (2015) Proteolytic Enzyme Combination Reduces Inflammation and Oxidative Stress and Improves Insulin Sensitivity in a Model of Metabolic Syndrome. Advances in Enzyme Research, 3, 1-8. 


\section{Introduction}

Metabolic Syndrome (MetS) is generally characterized by obesity and the presence of two additional metabolic risk factors [1]. More specifically, the risk factors encompassing MetS include abdominal obesity (waist circumference $\geq 40$ inches for men and $\geq 35$ inches for women), impaired fasting blood glucose ( $\geq 100 \mathrm{mg} / \mathrm{dL}$ ), elevated triglycerides ( $\geq 150 \mathrm{mg} / \mathrm{dL}$ ), low high-density lipoprotein (HDL $<40 \mathrm{mg} / \mathrm{dL}$ for men and $<50 \mathrm{mg} / \mathrm{dL}$ for women), and elevated blood pressure ( $\geq 130 / 85 \mathrm{mmHg}$ ) [1]. A public health crisis, MetS affects an estimated 20\% $34 \%$ of the population [2], increasing the risk for cardiovascular disease, stroke and diabetes and driving up medical costs. In the US alone, the health care costs associated with cardiovascular disease, obesity and diabetes are estimated at $\$ 444$ billion, \$147 billion, and \$218 billion, respectively [2]-[4]. Although largely preventable and modifiable by nutrition and lifestyle interventions, few pharmaceutical or nutritional agents are available to address the complex, multiple risk factors that contribute to MetS.

Emerging data suggest that MetS is associated with both inflammation and oxidative stress and that these may be independent risk factors for diabetes, hypertension and cardiovascular events, even after adjustment for established risk factors such as dyslipidemia [5]-[8]. It has further been proposed that C-reactive protein (CRP), as a marker of general inflammation, may be an independent predictor of long-term cardiovascular risk and that targeting elevated CRP might reduce the incidence of cardiovascular events [7] [9]-[10]. Statin therapy has been debated as a treatment for elevated CRP, independent of low-density lipoprotein (LDL) cholesterol levels and as primary prevention in patients at intermediate risk for heart disease [7] [9]. However, statin therapy is associated with myotoxicity and an increased incidence of diabetes [9].

Systemic Enzyme Therapy (SET) is the oral administration of combinations of proteolytic enzymes from plant sources, such as bromelain (Ananas comosus fruit) and papain (Carica papaya fruit); bovine or porcine pancreatic enzymes, such as trypsin, chymotrypsin, and pancreatin; and in some preparations, fungal-sourced lipase and amylase. Wobenzym ${ }^{\circledR}$ also includes the bioflavanoid rutin, which has been demonstrated to reverse and prevent metabolic changes such as abdominal fat stores, glucose tolerance, hepatic and cardiovascular function, as well as attenuate oxidative stress and inflammation in a rat model of MetS [11]. Systemic enzymes have a long history of use as natural anti-inflammatory agents, and such products are available commercially as dietary supplements or pharmaceuticals. A recent review summarizes the absorption and pharmacokinetics of orally administered proteolytic enzymes and provides the rationale behind the anti-inflammatory effects of enzymeantiprotease complexes [12]. Numerous animal [13]-[16] and human trials [17] [18] have demonstrated the clinical benefits of systemic enzyme therapy in inflammatory conditions, most commonly in comparative trials concluding non-inferiority to non-steroidal anti-inflammatory drugs (NSAIDs) [19], as well as in reducing general inflammation measured as C-reactive protein [20] [21]. However, to our knowledge, SET has not been studied in inflammation as related to parameters of MetS. Therefore, a pre-clinical trial was conducted to determine the effects of orally administered proteolytic enzymes on the prevention and treatment of inflammation, insulin sensitivity, oxidative stress, and other metabolic markers in an animal model of diet-induced MetS.

\section{Materials and Methods}

\subsection{Animals and Diets}

Male and female Chinchilla rabbits, aged 3 to 4 months, were obtained from the Ukranian Academy of Medical Sciences. The animal protocol was approved and housing provided by the National Scientific Center, ND Straschesko Institute of Cardiology in Kiev. The investigation included 50 rabbits with baseline body weights of $2.6 \pm$ $0.15 \mathrm{~kg}$ that were fed a regular diet supplemented with $0.75 \mathrm{~g} / \mathrm{kg}$ of body weight per day of dry milk cream containing $80 \%$ saturated fatty acids for 8 weeks with free access to water. The rabbits were separated into 3 groups: control group $(n=30)$, prevention group $(n=10)$, and treatment group $(n=10)$. Animals in the control group were fed the high-fat diet and received a saline vehicle control. Animals in the prevention group were fed the high-fat diet and administered Wobenzym ${ }^{\circledR}$ (Mucos Pharma GmbH, Oberhaching, Germany) for the full 8 weeks. Animals in the treatment group were fed the high-fat diet and administered Wobenzym from the 5th week to the 8th week. Wobenzym was dosed at 1 tablet per $6 \mathrm{~kg}$ of body weight, based on preliminary tests and equivalent to the typical human dose by weight. Tablets were ground and delivered in sterile saline directly into the small intestine via gastric tube connected to a Janne syringe. 


\subsection{Blood Collection and Parameters}

Blood samples were obtained at baseline and weeks 2, 4, 6, and 8 from the marginal ear vein, centrifuged at 4000 RPM, and processed immediately without freezing, as previously described [22]. The determinations of total cholesterol, trigylcerides (Tg), free fatty acids (FFA), blood glucose, glycosylated hemoglobin (HbA1c), and CRP were carried out using standard BioSystem test kits according to the manufacturer's protocols (Spain) and analyzed on a BTS-330 biochemical analyzer (Spain). Lipid oxidation was determined as the plasma malondialdehyde concentration using a standard reaction with thiobarbituric acid, TBARS (Sigma, USA).

\subsection{Insulin Sensitivity}

Systemic insulin sensitivity was determined as changes in plasma glucose level as a percentage of the initial value 60 min after subcutaneous insulin injection (Actrapid ${ }^{\circledR}$ HM, Novo Nordisk, Denmark), as previously described [23]. Results are reported as percent glucose clearance, calculated as follows:

$\%$ Glucose Clearance $=[($ plasma glucose at baseline $) /($ plasma glucose at $60 \mathrm{~min})] \times 100$.

\subsection{Statistical Analyses}

Data are presented as the mean \pm SEM. Analyses were performed using GraphPad InStat 3 software (La Jolla, CA). Comparisons between and within groups were made by analysis of variance (ANOVA) with Tukey-Kramer multiple comparisons. Mann-Whitney U tests are used when the data are not normally distributed. Significance was accepted at $P<0.05$.

\section{Results}

\subsection{Lipid Loading Induced Insulin Resistance and Metabolic Alterations}

Chronic lipid loading over 8 weeks induced a deterioration of systemic insulin sensitivity, determined as a reduction in percent glucose clearance following subcutaneous insulin injection (Figure 1(a), $P<0.001$ ). Lipid loading was also associated with increased inflammation (Figure 1(b), $P<0.001$ ), oxidative stress (Figure 1(c), $P<0.001$ ), HbA1c (Figure 1(h), $P<0.001$ ), and increased in lipid levels (total cholesterol, total TG and FFA levels (Figure 1(d)-(f), $P<0.001$ ) compared to baseline values.

\subsection{Systemic Enzyme Therapy Prevented the Decrease in Insulin Sensitivity}

Systemic insulin sensitivity was determined as changes in blood glucose levels as a percentage of the initial value at $60 \mathrm{~min}$ following subcutaneous insulin injection. At baseline, glucose clearance was $54.4 \% \pm 0.5 \%$, but in lipid-fed control animals this decreased to $8.0 \% \pm 0.9 \%$ at the 8th week (Figure 1(a)). The prevention and treatment groups maintained glucose clearance at $30.4 \% \pm 2.6 \%$ and $23.2 \% \pm 2.5 \%$, respectively, which differed from the control group at the 8 week $(P<0.001)$.

\subsection{Systemic Enzyme Therapy Reduced Diet-Induced Inflammation}

Lipid loading raised serum CRP throughout the study period from $1.75 \pm 0.11 \mathrm{mg} / \mathrm{L}$ at baseline to $13.6 \pm 0.74$ $\mathrm{mg} / \mathrm{L}$ and $32.03 \pm 2.22 \mathrm{mg} / \mathrm{L}$ in the control group at the 4th and 8th weeks, respectively (Figure 1(b)). At the 4th week, the increases in serum CRP in both the prevention $(10.1 \pm 1.4 \mathrm{mg} / \mathrm{L})$ and treatment $(13.4 \pm 2.49 \mathrm{mg} / \mathrm{L})$ groups were similar to control. At week 6, however, serum CRP levels in the prevention group (11.4 \pm 1.15 $\mathrm{mg} / \mathrm{L})$ but not in the treatment group $(19.2 \pm 2.54 \mathrm{mg} / \mathrm{L})$ were significantly lower from control group $(25.0 \pm 1.25$ $\mathrm{mg} / \mathrm{L})(P<0.001)$. Serum CRP decreased in week 8 compared to week 6 in both the prevention $(6.3 \pm 0.38$ $\mathrm{mg} / \mathrm{L})$ and treatment $(11.34 \pm 1.41 \mathrm{mg} / \mathrm{L})$ groups, and both groups differed from control $(P<0.001)$.

\subsection{Systemic Enzyme Therapy Prevented Oxidative Stress}

Oxidative stress, measured as serum malondialdehyde, increased in both the control and treatment groups as compared to baseline (Figure 1(c)). However, concurrent SET prevented the increase in oxidative stress observed with chronic lipid loading $(P<0.001)$.Treatment with SET initiated at the 5th week, did not affect oxida- 


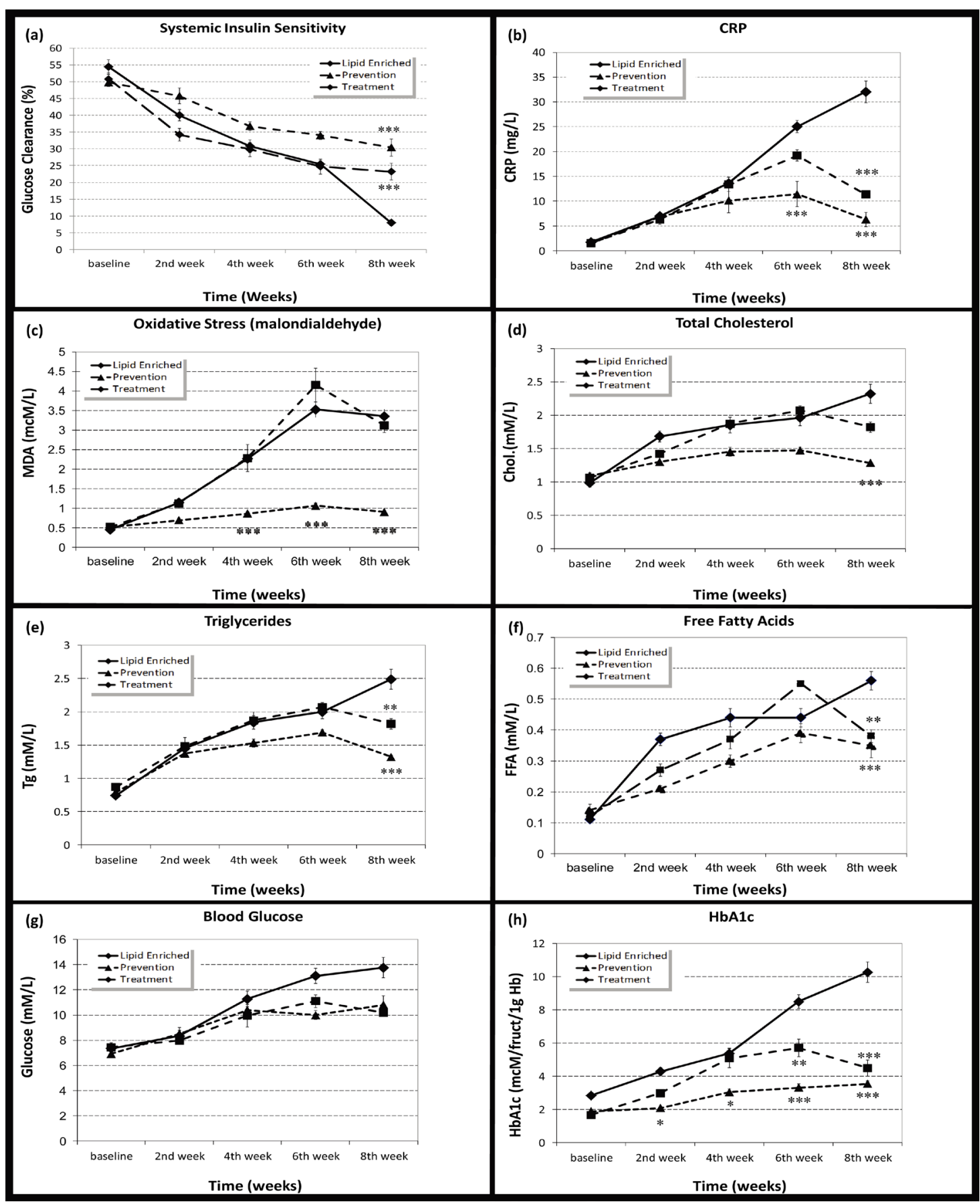

Figure 1. Effects of SET (Wobenzym ${ }^{\circledR}$ ) prevention (initiated at baseline) or treatment (initiated at 5 weeks) on parameters of MetS in chinchilla rabbits fed a lipid enriched diet. Values are mean $\pm \mathrm{SEM},{ }^{*} P<0.05,{ }^{* *} P<0.01,{ }^{* * *} P$ $<0.001$ compared to control group.

tive stress compared to the control group at 6 or 8 weeks.

\subsection{Systemic Enzyme Therapy Mediated Dyslipidemia}

Chronic lipid loading increased total cholesterol, Tg, and FFA in all groups compared to baseline (Figure 1(d)-(f)). Prevention and treatment groups did not differ from the control group for cholesterol, TG, and FFA through 6 weeks. At week 8, total cholesterol was reduced in the prevention group $(1.28 \pm 0.02 \mathrm{mmol} / \mathrm{L})$ com- 
pared to control group ( $2.32 \pm 0.14 \mathrm{mmol} / \mathrm{L}$ ) (Figure $1(\mathrm{~d}), P<0.001)$. Triglycerides were significantly lower in both the prevention $(1.32 \pm 0.03 \mathrm{mmol} / \mathrm{L}, P<0.001)$ and treatment $(1.82 \pm 0.08 \mathrm{mmol} / \mathrm{L}, P<0.01)$ groups compared to control $(2.49 \pm 0.15 \mathrm{mmol} / \mathrm{L}$ ) at week 8 (Figure 1(e)). Similarly, FFA were lower in the prevention $(P<0.001)$ and treatment $(P<0.01)$ groups compared to the control group at week 8 (Figure $1(\mathrm{f}))$. Free fatty acids appeared elevated in the treatment group at week 6 but this was not significant. In all three parameters, the treatment group appeared to follow the control group until week 6 (treatment initiated at week 5) and then declined relative to control.

\subsection{Systemic Enzyme Therapy Did Not Affect Blood Glucose but Reduced HbA1c}

Fasting blood glucose increased in response to lipid loading from $7.34 \pm 0.46 \mathrm{mmol} / \mathrm{L}$ at baseline to $13.8 \pm 0.81$ $\mathrm{mmol} / \mathrm{L}$ at the 8th week in the control group (Figure $1(\mathrm{~g})$ ). Although blood glucose levels appeared to be reduced in both the prevention $(10.8 \pm 0.74 \mathrm{mmol} / \mathrm{L})$ and the treatment $(10.2 \pm 0.27 \mathrm{mmol} / \mathrm{L})$ groups compared to control, the differences were not significant. However, while lipid loading significantly increased in HbA1c throughout 8 weeks in the control group, concurrent SET prevented the increase in HbA1c in the prevention group at all time points ( $3.54 \pm 0.17$ vs. $10.27 \pm 0.61 \mu$ mol fructose per $1 \mathrm{~g} \mathrm{Hb}$ at week $8, P<0.001$ ). Enzyme treatment initiated at week 5 also lowered HbA1c relative to control, observed at both the 6th $(P<0.01)$ and $8^{\text {th }}$ weeks (Figure 1(h), $P<0.001$ ).

\section{Discussion}

Hyperglycemia, impaired glucose disposal, hyperlipidemia, inflammation, and oxidative stress are central features of MetS. However, a limited etiological comprehension, together with a lack of consensus on qualitative and quantitative clinical definitions, has complicated the development and validation of an animal model that faithfully recapitulates human MetS.

While the most widely utilized MetS models are mice, rabbits offer a potentially valuable tool as they can depict a broader landscape of diet-induced MetS pathologies over a longer period of time. Their use in over 1000 studies of diabetes to date supports their basic utility in examining insulin sensitivity [24]. In response to fat and cholesterol feeding, rabbits develop the most well-established MetS features, including systemic inflammation, glucose intolerance, hyperlipidemia, hypertension, accrual of visceral fat tissue, and fatty liver disease [25]-[32]. Relative to mice, the lipoprotein profile of rabbits also corresponds more closely to humans, and in contrast to mice, which exhibit an atheroprotective phenotype, rabbits with a constellation of MetS features develop coronary atherosclerotic lesions resembling that of humans [24] [26]. In addition, as rabbits exhibit a longer life span of 5 - 8 years, lengthier study durations may afford more extensive interrogation of MetS and its complications in an easily handled, inexpensive animal model.

The Chinchilla rabbit employed in the current study corresponds, in baseline biochemical parameters, age, and approximate weight, with other rabbit models used to investigate MetS. Here, we report baseline fasting glucose, total cholesterol and Tg levels that are similar to published values from a recently developed rabbit model of high-fat diet (HFD)-induced MetS [25] [31] [32]. Baseline CRP values were similar to those reported in Japanese White rabbits utilized to investigate MetS [29]. The plasma response of glucose to insulin injection in the fasting state was consistent with published data using the same insulin preparation, dose and mode of administration [33].

The administration of SET, either as prevention beginning at baseline or as treatment beginning at the 5th week, failed to significantly reduce fasting glucose levels (Figure 1(g)). However, increased glucose clearance was evident in both groups (Figure 1(a)). Several potential reasons may account for this finding. The magnitude of improvement in insulin sensitivity may have been insufficient or of limited relevance to the fasting glucose level. Further, fasting glucose may have been somewhat refractory to the effects of insulin sensitization as a result of increased hepatic glucose production in the overnight fast. The lower HbA1c in SET-treated animals suggests that SET was attenuating blood glucose at time points other than the morning hours, such during the daytime postprandial periods.

While HbA1c is universally employed to assess long-term glycemic control in humans, considerable species differences exist, and a paucity of literature addresses its ranges in rabbits. In the current study, HbA1c was lowest in the SET prevention group, with significance demonstrated at the 2nd week (Figure 1(h)). The superior efficacy in the prevention group, which was of longer duration than the treatment approach, is consistent with 
the cumulative nature of this marker. The most striking finding was the reduction in HbA1c at the 6th week in the treatment group, which received the first dose of SET at the 5th week. This rapid change occurred despite no concomitant alteration of systemic insulin sensitivity or fasting blood glucose. It is clear that in our rabbit model, this marker is subject to change modification within a short period of time.

Elevated CRP reflects systemic inflammation, a highly pertinent feature of MetS. In rabbits, this marker is inducible by stimuli such as dietary lipid enrichment [29] and is repressed by anti-inflammatory and anti-hyperglycemic interventions [27]. Like humans, rabbits exhibit elevations of CRP in tandem with progression of MetS features including atherosclerosis [29]. We demonstrate an approximate 10-fold increase in response to a HFD (Figure 1(b)), which is similar to previously reported data in rabbits that developed other MetS features, including visceral adiposity and atherogenic lesions, over 22 weeks of HFD feeding [29].

Malondialdehyde (MDA), a thoroughly validated marker of oxidative stress, increased 7-fold over 8 weeks (Figure 1(c)). Since proteases are not recognized as potent antioxidants, it is rational to speculate that SET may act indirectly through reduction of inflammation-associated reactive oxygen species generation, and/or the effect is due to the co-formulation with rutin, a flavonoid antioxidant with potential utility in MetS [34]. The absence of a therapeutic effect may be explained by oxidative stress comprising an early, initiating event in MetS. As a nonsignificant decrease was apparent in the treatment group between the 6th and 8th week, continuation of SET over a longer duration may be necessary for antioxidant effects to become detectable.

The 3-fold elevation of Tg in untreated rabbits was similar to the 3.5-fold increase in another MetS HFD-fed rabbit model after 12 weeks (Figure 1(e)) [25]. The SET as a preventive or treatment measure resulted in lower Tg levels very late in the study. A late reduction, although significant only in the prevention group, was also evident in total cholesterol levels. In untreated animals, elevation of total cholesterol in our model was modest compared to other studies of HFD-fed rabbits [25] [28]. For both Tg and cholesterol levels, SET as a prevention produced the lowest values throughout the study, while treatment had a relatively weak effect (Figure 1(d)). The short duration of treatment with SET (beginning at the 5th week, with analysis at the 8th week) was likely insufficient, and the nonsignificant decline suggests that continuation may have produced further reductions. Previous studies of MetS in rabbits have administered the HFD over a longer time period of 12 - 22 weeks [25] [28] [29] [32].

Several possible explanations may account for the general observation of superior efficacy of prevention relative to treatment. Foremost, the longer duration of supplementation in the prevention group may account for the superior efficacy. A second possibility is that SET exerts its effects in the early, initiating stages of MetS, as oxidative events and inflammatory signals are causally involved in the onset of insulin resistance [35]. A third possibility is that visceral adiposity, although not assessed, is likely to have increased as described previously [25] [29] [32], increasing oxidative stress and inflammation in the first weeks of high-fat diet to a point that requires a higher dose of SET [29] [36]. Alternatively, late commencement of SET may have been insufficient to address a modified climate of substantial and multifocal metabolic deregulation. From a theoretical perspective, a therapy that is effective when introduced at a later stage of disease must target pathways of reversal or attenuation, and these may differ from pathways of causation. Accordingly, preventive agents are not always curative, and vice versa.

While previous research has documented the bioavailability and therapeutic efficacy of SET as an anti-inflammatory intervention, there is limited knowledge of its mechanisms of action [12] [13] [37]. It has been demonstrated that these proteases, as complexes with endogenous antiproteases, complex with cytokines and promote their clearance from inflamed tissue via endocytotic and phagocytic routes [13]. Other mechanisms, such as interactions with cell surface receptors and signal transduction events, have been suggested [13]. In contrast to more extensively studied natural anti-inflammatory agents, such as fish oil and polyphenols, which intersect established pathways with known relevance to MetS, it remains unknown how proteolytic enzymes relate to the onset and progression of MetS.

\section{Conclusion}

The current data contribute to an evolving body of literature supporting the potential of anti-inflammatory interventions in MetS prevention and therapy. A continuation of this work is clearly warranted, including validation of this potentially valuable animal model in the study of MetS. Wobenzym ${ }^{\circledR}$ improved insulin sensitivity and reduced CRP and other risk factors associated with MetS in this model. Further research is required to determine 
whether anti-inflammatory interventions, and SET in particular, might affect MetS development and progression in humans.

\section{Acknowledgements}

The authors thank Barry W. Ritz, PhD, Kelly Heim, PhD, and Kelly Gibson for their assistance in preparing the manuscript for publication, including adaptation to English.

\section{References}

[1] Alberti, K.G., Zimmet, P. and Shaw, J. (2005) The Metabolic Syndrome-A New Worldwide Definition. The Lancet, 366, 1059-1062. http://dx.doi.org/10.1016/S0140-6736(05)67402-8

[2] Goldstein, L.B., Bushnell, C.D., Adams, R.J., Appel, L.J., Braun, L.T., Chaturvedi, S., Creager, M.A., Culebras, A., Eckel, R.H., Hart, R.G., Hinchey, J.A., Howard, V.J., Jauch, E.C., Levine, S.R., Meschia, J.F., Moore, W.S., Nixon, J.V., Pearson, T.A., American Heart Association Stroke Council, Council on Cardiovascular Nursing, Council on Epidemiology and Prevention, Council for High Blood Pressure Research, Council on Peripheral Vascular Disease, and Interdisciplinary Council on Quality of Care and Outcomes Research (2011) Guidelines for the Primary Prevention of Stroke: A Guideline for Healthcare Professionals from the American Heart Association/American Stroke Association. Stroke, 42, 517-584. http://dx.doi.org/10.1161/STR.0b013e3181fcb238

[3] Finkelstein, E.A., Trogdon, J.G., Cohen, J.W. and Dietz, W. (2009) Annual Medical Spending Attributable to Obesity: Payer- and Service-Specific Estimates. Health Affairs (Millwood), 28, W822-W831. http://dx.doi.org/10.1377/hlthaff.28.5.w822

[4] Center for Disease Control and Prevention, National Center for Chronic Disease Prevention and Health Promotion. (2011) National Diabetes Fact Sheet. http://www.cdc.gov/diabetes/pubs/pdf/ndfs_2011.pdf

[5] Holvoet, P., Lee, D.-H., Steffes, M., Gross, M. and Jacobs, D.R. (2008) Association between Circulating Oxidized Low-Density Lipoprotein and Incidence of the Metabolic Syndrome. The Journal of the American Medical Association, 299, 2287-2293. http://dx.doi.org/10.1001/jama.299.19.2287

[6] Sun, S.Y., Ji, Y.W., Kersten, S. and Ling, Q. (2012) Mechanisms of Inflammatory Responses in Obese Adipose Tissue. Annual Review of Nutrition, 32, 261-286. http://dx.doi.org/10.1146/annurev-nutr-071811-150623

[7] Deveraj, S., Siegel, D. and Jialal, I. (2011) Statin Therapyin Metabolic Syndrome and Hypertension Post-JUPITER: What Is the Value of CRP? Current Atherosclerosis Reports, 13, 31-42. http://dx.doi.org/10.1007/s11883-010-0143-2

[8] Den Englensen, C., Koekkoek, P.S., Gorter, P.J., van den Donk, M., Salomé, P.L. and Rutten, G.E. (2012) High-Sensitivity C-Reactive Protein to Detect Metabolic Syndrome in a Centrally Obese Population: A Cross-Sectional Analysis. Cardiovascular Diabetology, 11, 25. http://dx.doi.org/10.1186/1475-2840-11-25

[9] Kones, R. (2010) Rosuvastatin, Inflammation, C-Reactive Protein, JUPITER, and Primary Prevention of Cardiovascular Disease-A Perspective. Drug Design, Development and Therapy, 4, 383-413. http://dx.doi.org/10.2147/DDDT.S10812

[10] Ridker, P.M. (2004) High-Sensitivity C-Reactive Protein, Inflammation, and Cardiovascular Risk: From Concept to Clinical Practice to Clinical Benefit. American Heart Journal, 148, S19-S26. http://dx.doi.org/10.1016/j.ahj.2004.04.028

[11] Panchal, S.K., Poudyal, H., Arumugam, T.V. and Brown, L. (2011) Rutin Attenuates Metabolic Changes, Nonalcoholic Steatohepatitis, and Cardiovascular Remodeling in High-Carbohydrate, High-Fat Diet-Fed Rats. Journal of Nutrition, 141, 1062-1069. http://dx.doi.org/10.3945/jn.111.137877

[12] Lorkowski, G. (2012) Gastrointestinal Absorption and Biological Activities of Serine and Cysteine Proteases of Animaland Plant Origin: Review on Absorption of Serine and Cysteine Proteases. International Journal of Physiology and Pathophysiology, 4, 10-27.

[13] Rovenská, E., Svík, K., Stančíková, M. and Rovenský, J. (1999) Enzyme and Combination Therapy with Cyclosporin A in the Rat Developing Adjuvant Arthritis. International Journal of Tissue Reactions, 21, 105-111.

[14] Rovenská, E., Svík, K., Stančíková, M., and Rovenský, J. (2001) Inhibitory Effect of Enzyme Therapy and Combination Therapy with Cyclosporin A on Collagen-Induced Arthritis. Clinical and Experimental Rheumatology, 19, 303- 309.

[15] Singer, F. and Oberleitner, H. (1996) Ein Beitrag zur medikamentösen Therapie der aktivierten Arthrose. Zur Effektivität eines Enzymgemisches versus Diclofenac. Wiener Meidizinische Wochenschrift, 3, 55-58.

[16] Skutelis, A., Pule, E., Purvins, I., Baltkajs, J., Skromanis, M., Zeibarts, M., Desser. L., Osterreicher, J. and Ansberg, J. (2001) Phlogenzym May Prevent Metabolic Injuries to Myocardium Results of a Laboratory Experiment with Rats. International Journal of Immunotherapy, 17, 59-64.

[17] Duskova, M. and Wald, M. (1999) Orally Administered Proteases in Aesthetic Surgery. Aesthetic Plastic Surgery, 23, 41-44. http://dx.doi.org/10.1007/s002669900241 
[18] Klein, G., Kullich, W. and Schwann, H. (1995) Double-Blind Trial of an Enzyme Therapy versus Oral Gold in Rheumatoid Arthritis. Clinicum, 3, 2-8.

[19] Kleine, M.W. (1990) Systemische enzymtherapie in der sportmedizin-grundlagen, wirkmechanismen, indikationen. Deutsche Zeitschriftfür Sportmedizin, 41, 126-134.

[20] Vinzenz, K. (1991) Ödembehandlung bei zahnchirurgischen eingriffen mit hydrolytischen enzymen. Chirurgische Zahnheilkunde, 7, 1053-1064.

[21] Kasseroller, R. and Wenning, H.G. (2003) Efficacy and Tolerability of Proteolytic Enzymes as an Anti-Inflammatory Agent in Lymphoedema after Axillary Dissection due to Mammary Cancer. The European Journal of Lymphology, 37-38, 18-26.

[22] Diehl, K.-H., Hull, R., Morton, D., Pfister, R., Rabemampianina, Y., Smith, D., Vidal, J.-M. and van de Vorstenbosch, C. (2001) A Good Practice Guide to the Administration of Substances and Removal of Blood, Including Routes and Volumes. Journal of Applied Toxicology, 21, 15-23. http://dx.doi.org/10.1002/jat.727

[23] Arakawa, Y., Kurokawa, N., Maeda, Y. and Yanaihara, C. (1989) Change in Solubility of Insulin in Therapeutic Mixtures of Insulin Preparations: HPLC Analysis. Diabetes Research and Clinical Practice, 7, 93-100. http://dx.doi.org/10.1016/0168-8227(89)90094-6

[24] Kainuma, M., Fujimoto, M., Sekiya, N., Tsuneyama, K., Cheng, C., Takano, Y., Terasawa, K. and Shimada, Y. (2006) Cholesterol-Fed Rabbit as a Unique Model of Nonalcoholic, Nonobese, Non-Insulin-Resistant Fatty Liver Disease with Characteristic Fibrosis. Journal of Gastroenterology, 41, 971-980. http://dx.doi.org/10.1007/s00535-006-1883-1

[25] Zhao, S., Chu, Y., Zhang, C., Lin, Y., Xu, K., Yang, P., Fan, J. and Liu, E. (2008) Diet-Induced Central Obesity and Insulin Resistance in Rabbits. Journal of Animal Physiology and Animal Nutrition, 92, 105-111.

[26] Li, H., Dai, M., and Jia, W. (2009) Paeonol Attenuates High-Fat-Diet-Induced Atherosclerosis in Rabbits by Anti-Inflammatory Activity. Planta Medica, 75, 7-11. http://dx.doi.org/10.1055/s-0028-1088332

[27] Zheng, H., Zhang, C., Yang, W., Wang, Y., Lin, Y., Yang, P., Yu, Q., Fan, J. and Liu, E. (2009) Fat and Cholesterol Diet Induced Lipid Metabolic Disorders and Insulin Resistance in Rabbit. Experimental and Clinical Endocrinology \& Diabetes, 117, 400-405. http://dx.doi.org/10.1055/s-0028-1102918

[28] Waqar, A.B., Koike, T., Yu, Y., Inoue, T., Aoki, T., Liu, E. and Fan, J. (2010) High-Fat Diet without Excess Calories Induces Metabolic Disorders and Enhances Atherosclerosis in Rabbits. Atherosclerosis, 213, 148-155. http://dx.doi.org/10.1016/j.atherosclerosis.2010.07.051

[29] Varga, O., Harangi, M., Olsson, I.A. and Hansen, A.K. (2010) Contribution of Animal Models to the Understanding of the Metabolic Syndrome: A Systematic Overview. Obesity Reviews, 11, 792-807. http://dx.doi.org/10.1111/j.1467-789X.2009.00667.x

[30] Morelli, A., Comeglio, P., Filippi, S., Sarchielli, E., Cellai, I., Vignozzi, L., Yehiely-Cohen, R., Maneschi, E., Gacci, M., Carini, M., Adorini, L., Vannelli, G.B. and Maggi, M. (2012) Testosterone and Farnesoid X Receptor Agonist INT-747 Counteract High Fat Diet-Induced Bladder Alterations in a Rabbit Model of Metabolic Syndrome. The Journal of Steroid Biochemistry and Molecular Biology, 132, 80-92. http://dx.doi.org/10.1016/j.jsbmb.2012.02.007

[31] Maneschi, E., Morelli, A., Filippi, S., Cellai, I., Comeglio, P., Mazzanti, B., Mello, T., Calcagno, A., Sarchielli, E., Vignozzi, L., Saad, F., Vettor, R., Vannelli, G.B. and Maggi, M. (2012) Testosterone Treatment Improves Metabolic Syndrome-Induced Adipose Tissue Derangements. Journal of Endocrinology, 215, 347-362. http://dx.doi.org/10.1530/JOE-12-0333

[32] Vignozzi, L., Morelli, A., Sarchielli, E., Comeglio, P., Filippi, S., Cellai, I., Maneschi, E., Serni, S., Gacci, M., Carini, M., Piccinni, M.P., Saad, F., Adorina, L., Vannelli, G.B. and Maggi, M. (2012) Testosterone Protects from Metabolic Syndrome-Associated Prostate Inflammation: An Experimental Study in Rabbit. Journal of Endocrinology, 212, 71-84. http://dx.doi.org/10.1530/JOE-11-0289

[33] Wang, J., Wan, R., Mo, Y., Zhang, Q., Sherwood, L.C. and Chien, S. (2010) Creating a Long-Term Diabetic Rabbit Model. Experimental Diabetes Research, 2010, Article ID: 289614. http://dx.doi.org/10.1155/2010/289614

[34] Hirabara, S.M., Gorjão, R., Vinolo, M.A., Rodrigues, A.C., Nachbar, R.T. and Curi, R. (2012) Molecular Targets Related to Inflammation and Insulin Resistance and Potential Interventions. Journal of Biomedicine and Biotechnology, 2012, Article ID: 379024. http://dx.doi.org/10.1155/2012/379024

[35] Lear, S.A., Sarna, L.K., Siow, T.J., Mancini, G.B., Siow, Y.L. and O, K. (2012) Oxidative Stress Is Associated with Visceral Adipose Tissue and Subclinical Atherosclerosis in a Healthy Multi-Ethnic Population. Applied Physiology, Nutrition, and Metabolism, 37, 1164-1170. http://dx.doi.org/10.1139/h2012-107

[36] Levenets, V.M., Lopushan, V.M., Terzov, A.I., Kharytonov, O.D. and Khindi, M. (1997) Systemic Enzyme Therapy in Orthopedics and Traumatology. Klinichna Khirurhiia, No. 9-10, 50-51.

[37] Siniachenko, O.V., Kovalenko, V.N., Mukhin, I.V., Ignatenko, G.A. and Terzov, A.I. (2000) Systemic Enzyme Therapy in Chronic Glomerulonephritis. Terapevticheskiu Arkhiv, 72, 46-48. 